\title{
Study of the reference standard facility of rhodium-iron resistance thermometer
}

\author{
L. Yin \\ School of Science, Beijing University of Chemical Technology, Beijing 100029, China \\ E-mail: yinliang@mail.buct.edu.cn \\ P. Lin
}

Technical Institute of Physics and Chemistry, Chinese Academy of Sciences, Beijing 100190, China

E-mail: linp@nim.ac.cn

X. Qi

School of Science, Beijing University of Chemical Technology, Beijing 100029, China

E-mail: qixin@mail.buct.edu.cn

Received June 21, 2013, revised September 2, 2013

\begin{abstract}
A 704 type ${ }^{4} \mathrm{He}$ cryostat is designed and the "National (China) Temperature Working Standard Group of the ITS-90 at 1.2-24 K" have been composed. A 703 type ${ }^{3} \mathrm{He}$ cryostat have been established and the working temperature of the "National Temperature Working Standard Group of the ITS-90 at 1.2-24 K" is extended to $0.65 \mathrm{~K}$, which is the lowest limit temperature of ITS-90. The 5 rhodium-iron resistance thermometers (RIRTs) which compose the Working Standard Group have been transformed to the International Temperature Scale of 1990 (ITS-90). A set of indexing data is fitted and calculated as the indexing table of the standard facility of RIRT. 42 basis temperature points have been measured at $0.67-26.37 \mathrm{~K}$. The results show that the temperature control level could reach $0.5 \mathrm{mK} / 30 \mathrm{~min}$. The difference between the temperature value of the 5 RIRTs and the average is less than $1.1 \mathrm{mK}$, and the comparison measurements uncertainty is $0.95 \mathrm{mK}$.
\end{abstract}

PACS: 06.20.fb Standards and calibration.

Keywords: rhodium-iron resistance thermometer, working standard, cryostat, temperature measurement.

\section{Introduction}

The International Temperature Scale of 1990 (ITS-90) stipulate that the International Kelvin temperature $\left(T_{90}\right)$ is divided into three temperature ranges to define below $273.16 \mathrm{~K}$ [1]. The temperature between $0.65-5 \mathrm{~K}$ is defined by ${ }^{3} \mathrm{He}$ and ${ }^{4} \mathrm{He}$ vapor pressure thermometer. It is defined by the interpolating constant volume gas thermometer of helium at 3.0-24.5561 K. At 13.8033-273.16 K temperature zone, platinum resistance thermometer is used as the inserted instrument, a set of defined fixed points and a specified interpolation method are used to define the temperature value between the fixed-point. After the promulgation and implementation of ITS-90, only the United States National Institute of Standards and Technology (NIST) and the German Federal Physical Technical Institute (PTB) reproduce vapor pressure thermometer and inserted gas thermometer at 0.65-24.5561 K temperature zone in accordance with the requirements of the Interna- tional Temperature Scale. The temperature scale between 0.5-27.1 K in Canada, France, Italy, Australia and other countries' metrology laboratory are all saved by the standard RIRTs of the United Kingdom National Physical Laboratory (NPL) temperature scale, which is translated to the $T_{90}$ by the temperature scale difference between the old and the new. The most important reason is that the standard RIRT is easy to be used and it has excellent long-term stability [2]. Rusby introduced that the apparent change of RIRT is less than $0.4 \mathrm{mK}$ in eight and a half years [3-6]. Lin Peng introduced the stability of RIRT can be achieved $0.1 \mathrm{mK}$ in 10 years [7-11].

Zhang Guo Quan used comparison method to establish "National Temperature Working Standard Group of the ITS-90 at 1.2-24 K" with 5 NPL RIRTs in China Institute of Metrology. The uncertainty is $2.7 \mathrm{mK}$. Due to the limitations of the early cryostat and the temperature control device, the earlier measurement data quality is not ideal. Therefore, it is important to improve the performance of 
the device for comparison measurements at the $1.2-27 \mathrm{~K}$ temperature zone, enhance the reliability of the measurement data and improve the conditions for keeping and dissemination of the value of temperature quantity.

We have designed A new 704 type ${ }^{4}$ He cryostat. A precision temperature control device and an automatic bridge are used to establish the reference standard facility of RIRT which improve the level of the dissemination of the temperature quantity at $1.2-27 \mathrm{~K}$. A 703 type ${ }^{3}$ He cryogenic is established which extended the working temperature of the "National Temperature Working Standard Group of the ITS-90 at $1.2-24 \mathrm{~K}$ ” to $0.65 \mathrm{~K}$ which is the lower limit temperature of ITS-90.

\section{Structural analysis of the reference standard facility of rhodium-iron resistance thermometer}

\subsection{4 type ${ }^{4}$ He cryostat}

Cryostat is an important part of the standard facility of rhodium-iron resistance thermometer. The design principles and manufacturing process of the cryostat influence the measurement level directly. A 704 type ${ }^{4}$ He cryostat is designed based on the design principles of the Australian National Measurement Laboratory (NML), as shown in Fig. 1. The outside and inside radiation screens are used to reduce the impact of the radiation heat leakage on the measurement results. The outside radiation screen which fixed below the $1 \mathrm{~K}$ pot reaches a consistent temperature with the $1 \mathrm{~K}$ pot by heat conduction. The inside radiation shield which fixed at the bottom of the constant temperature copper block is formed of two semi-cylindrical screens with end caps and all RIRTs are wrapped in it. The temperature control element is not used for the inside radiation shield and the inside radiation shield reaches a consistent tempe-

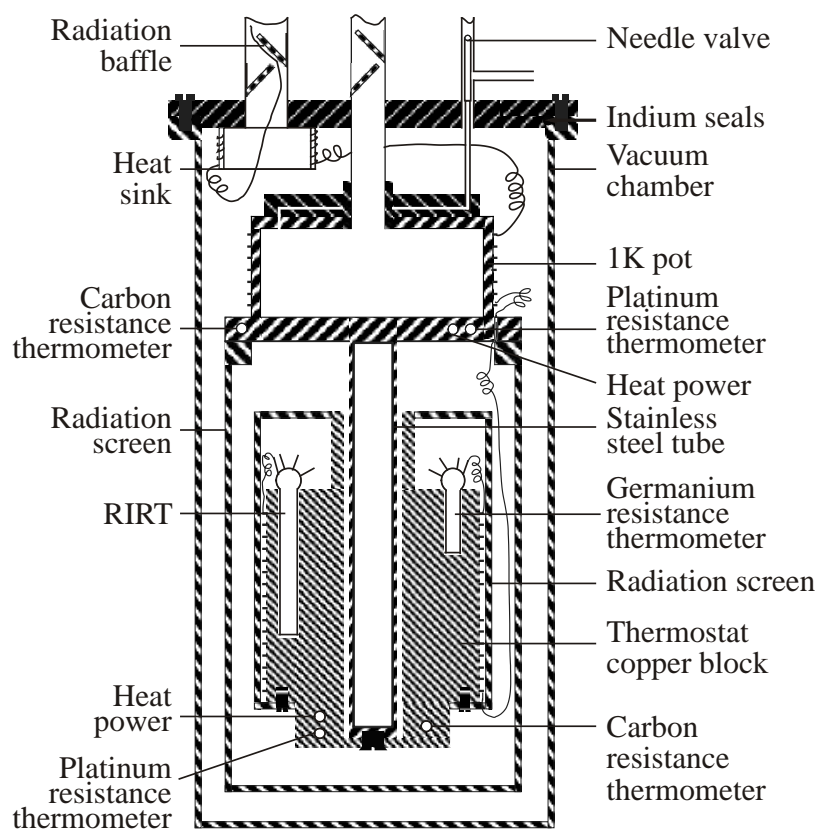

Fig. 1. Schematic illustration of the 704 type cryostat. rature with the constant temperature copper block through heat conduction. The measurement and control leads of the 704 type cryostat do thermal anchor at the vacuum chamber heat sink, outer wall of the $1 \mathrm{~K}$ pot and the outer wall of the constant temperature copper block. The lead between the $1 \mathrm{~K}$ pot and the constant temperature copper block is lengthened to minimize the interference to the constant temperature copper block by the heat leak along the measurement leads. The temperature of the constant temperature copper block is under control when comparison measurements is performed below $5 \mathrm{~K}$. When comparison measurements is conducted above $5 \mathrm{~K}$, the temperature of the $1 \mathrm{~K}$ pot and the constant temperature copper block are all controlled to minimize the inhomogeneity of the temperature field of the copper block.

\subsection{3 type ${ }^{3}$ He cryostat}

The ${ }^{3}$ He cryostat is used to obtain the temperature below $1.2 \mathrm{~K}$. The core of the 703 type ${ }^{3} \mathrm{He}$ cryostat is made by Oxford Instruments. The 703 type ${ }^{3} \mathrm{He}$ cryostat is activated carbon adsorption and inner decompression mode. There are 10 liters standard state $\left(0{ }^{\circ} \mathrm{C}, 1 \text { bar }\right)^{3} \mathrm{He}$ gas as the annex to the 703 type ${ }^{3} \mathrm{He}$ cryostat. The system block diagram of 703 type cryostat is showed in Fig. 2.

The work temperature of 703 type cryostat is $0.5-5 \mathrm{~K}$. In this temperature the radiation heat leak could be ignored, so the radiation shield is not installed. The heating power is small and the thermal diffusion rate of the copper is high, so a single temperature control is used at the copper block. The 703 type cryostat gets $4.2 \mathrm{~K}$ with liquid ${ }^{4} \mathrm{He}$. Then the $1 \mathrm{~K}$ pot of the 703 type cryostat is pumped to cool and liquefy ${ }^{3} \mathrm{He}$ gas to less than $2 \mathrm{~K}$. The liquid ${ }^{3} \mathrm{He}$ is cooled by pumping the activated carbon adsorption to the lowest temperature, then the constant temperature copper block is heated and controlled to carry out the experiments.

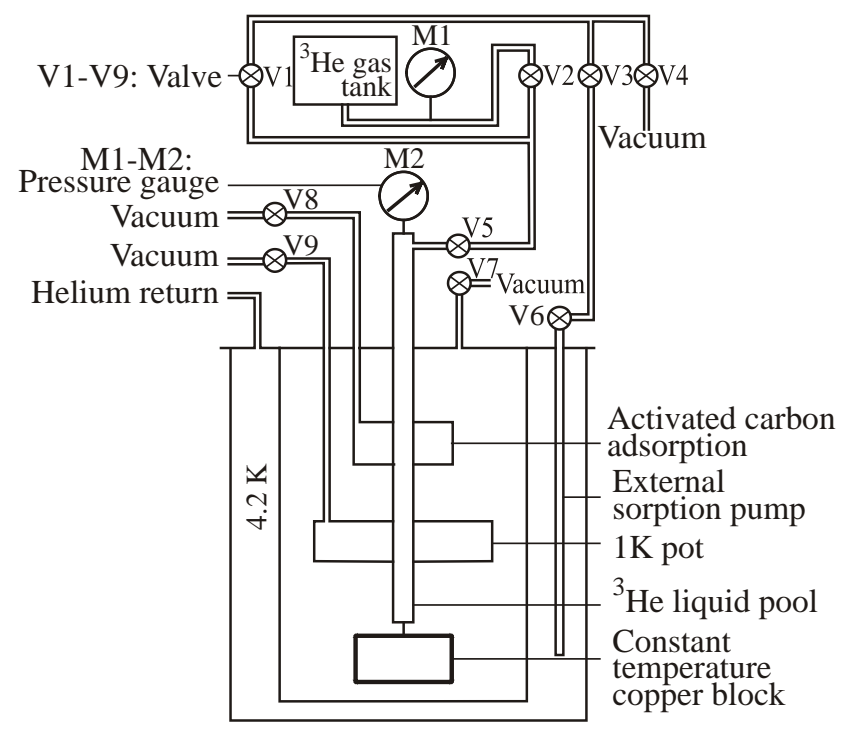

Fig. 2. System diagram of the 703 type ${ }^{3}$ He cryostat. 
Table 1. Main parameters of the 5 RIRTs

\begin{tabular}{c|c|c|c|c}
\hline \hline $\begin{array}{c}\text { Thermometer serial } \\
\text { number }\end{array}$ & $\begin{array}{c}\text { Calibrating points } \\
\text { number }\end{array}$ & $\begin{array}{c}\text { Resistance at 4.22 K, } \\
\Omega\end{array}$ & $\begin{array}{c}\text { Resistance with temperature } \\
\text { ratio at 4.22 K, } \Omega / \mathrm{mK}\end{array}$ & Calibrating date \\
\hline \hline 29822 & 47 & 4.540 & 3.23 & 3.58 \\
A19 & 37 & 3.842 & 3.98 & 1977 \\
A20 & 37 & 3.605 & 5.26 & 1980 \\
A34 & 34 & 2.827 & 4.83 & 1983 \\
A35 & 34 & 3.070 & 1983 \\
\hline \hline
\end{tabular}

\subsection{RIRT temperature working standard group}

The reference standard facility of RIRT is composed of $5 \mathrm{Rh}-\mathrm{Fe}$ resistance thermometers. The five RIRTs are made by Tinsley and calibrated by NPL in accordance with 0.5-30 K Temporary International Temperature Scale "EPT-76" at 0.5-27.1 K. The measurement uncertainty is $0.2 \mathrm{mK}$ and the calibration uncertainty is $1 \mathrm{mK}$. The main parameters of the 5 RIRTs calibrated by NPL is showed in Table 1.

The 5 RIRTs are calibrated by NPL in accordance with EPT-76, so the RIRTs should be converted to the ITS-90 according to ITS-90 text. The temperature difference between ITS-90 and EPT-76 is showed in Table 2. The international electrical unit is changed at 1990, which have the most impact on RIRT with $0.1 \mathrm{mK}$ at $27 \mathrm{~K}$. The calibration table of the RIRTs are fitted and calculated based on the British NPL indexing data.

Table 2. The temperature difference between ITS-90 and EPT-76.

\begin{tabular}{c|c|c|c}
\hline \hline Temperature, $\mathrm{K}$ & $T_{90}-T_{76}, \mathrm{mK}$ & Temperature, $\mathrm{K}$ & $T_{90}-T_{76}, \mathrm{mK}$ \\
\hline \hline 1 & 0 & 15 & -1.3 \\
2 & 0 & 16 & -1.4 \\
3 & 0 & 17 & -1.6 \\
4 & 0 & 18 & -1.8 \\
5 & -0.1 & 19 & -2.0 \\
6 & -0.2 & 20 & -2.2 \\
7 & -0.3 & 21 & -2.5 \\
8 & -0.4 & 22 & -2.7 \\
9 & -0.5 & 23 & -2.0 \\
10 & -0.6 & 24 & -3.2 \\
11 & -0.7 & 25 & -3.5 \\
12 & -0.8 & 26 & -3.8 \\
13 & -1.0 & 27 & -4.1 \\
14 & -1.1 & \multicolumn{3}{|l}{} \\
\hline \hline
\end{tabular}

\subsection{Experimental devices}

The system block diagram of 704 type cryostat is shown in Fig. 3. The temperature of the $1 \mathrm{~K}$ pot and the constant temperature copper block are controlled. The re-

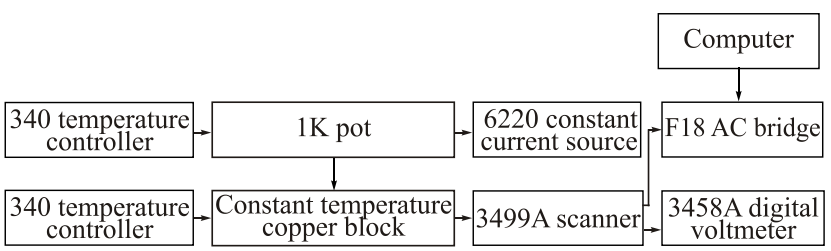

Fig. 3. Schematic illustration of the 704 type cryostat. sistance of the RIRTs and the germanium resistance thermometers are measured.

The system block diagram of 703 type cryostat is showed in Fig. 4. The temperature of the constant temperature copper block is controlled and the resistance of the RIRTs and germanium resistance thermometers are measured.

The temperature of the constant temperature copper block is controlled by the Lake Shore 340 Temperature Controllers. The lowest temperature for controlled and measured by the 340 Temperature Controllers is $0.1 \mathrm{~K}$ and the temperature display accuracy is $0.1 \mathrm{mK}$ below $10 \mathrm{~K}$ and is $1 \mathrm{mK}$ above $10 \mathrm{~K}$. The ASL F18 AC bridge is used to measure the resistance of the RIRTs. The resistance resolution of the F18 AC bridge is $1 \mu \Omega$ with a $10 \Omega$ standard resistor, which is equivalent to a temperature resolution of $3 \mu \mathrm{K}$. The measurement accuracy of the F18 AC bridge is highest when the measurement bandwidth is set at $0.02 \mathrm{~Hz}$, and the corresponding sampling time is 50 seconds. When the measurement bandwidth of the F18 AC bridge is set at $0.1 \mathrm{~Hz}$, the corresponding sampling time is 10 seconds, but the divergence of the measurement results will increase.

The RIRT A34 is used as the primary thermometer to record the temperature. Every measurement point is measured three times with RIRT A34, and another two RIRTs are used to measure the temperature in the middle of each time. The average value of the three measurements of RIRT A34 is used as the last measurement value.

\section{Result and discussion}

\subsection{Temperature control curve of RIRTs}

The temperature control ability of the cryostat determines the quality of the RIRT measurement and it is determined by the uniformity, stability and fluctuation of the temperature field of the copper block as well as the interference caused by the measurement conversion of the electrical bridge. Fig. 5 shows the temperature control curve of the reference standard facility of RIRTs at 4.5, 14.5 and $23.4 \mathrm{~K}$.

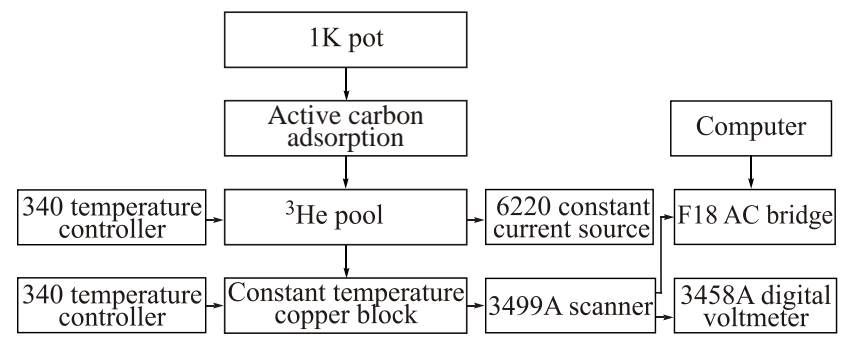

Fig. 4. Schematic illustration of the 703 type cryostat. 

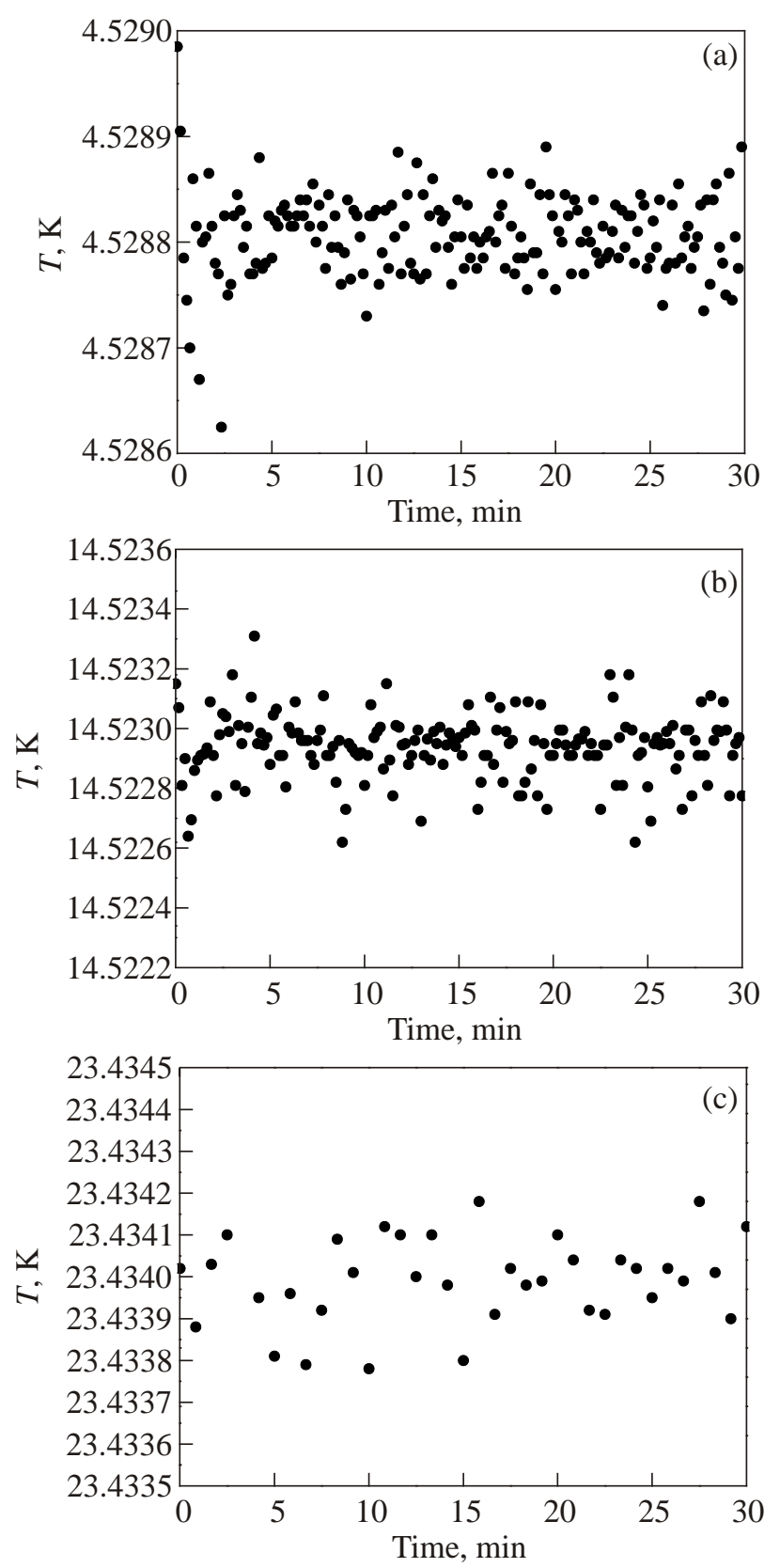

Fig. 5. The temperature control curve at T, K: 4.5 (a), 14.5 (b), 23.4 (c).

The measurement bandwidth of F18 AC bridge is set at $0.1 \mathrm{~Hz}$ when $4.5 \mathrm{~K}$ and $14.5 \mathrm{~K}$ is measured and $0.02 \mathrm{~Hz}$ is set at $23.4 \mathrm{~K}$. The temperature fluctuation is about $0.2 \mathrm{mK} / 30 \mathrm{~min}$ at $4.5 \mathrm{~K}$ as showed in Fig. 5(a). The temperature fluctuation is about $0.5 \mathrm{mK} / 30 \mathrm{~min}$ at $14.5 \mathrm{~K}$ and $0.4 \mathrm{mK} / 30 \mathrm{~min}$ at $23.4 \mathrm{~K}$ as showed in Fig. 5(b) (c).

\subsection{Measurement results}

The 5 RIRTs is used for the comparison measurements at 42 temperature points. The relationship between the measurements of the 5 RIRTs and the average value is showed in Fig. 6. The difference between the measurements of the 5 RIRTs and the average value is less than $1.1 \mathrm{mK}$.

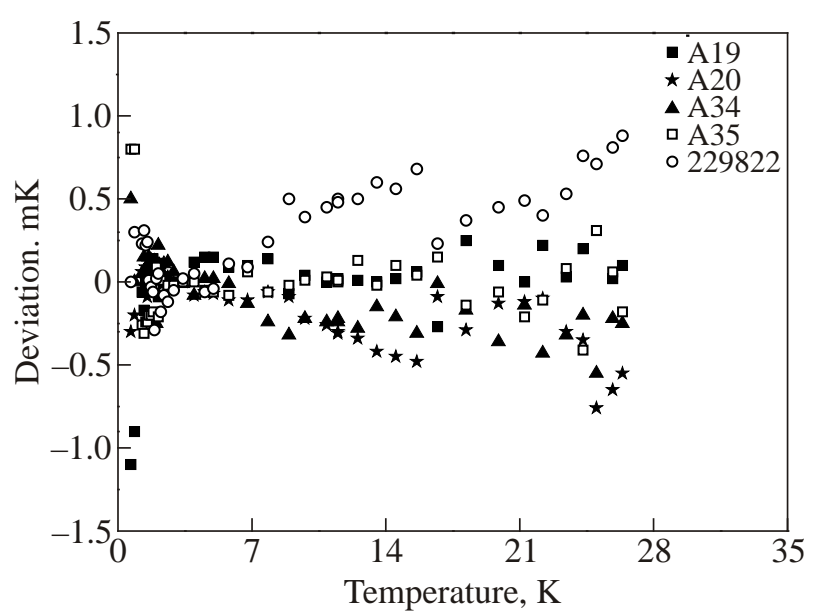

Fig. 6. The relationship between the measurements of the 5 RIRTs and the average value.

\subsection{Measurement uncertainty analysis}

The uncertainty of the comparison measurement includes the following components:

NPL indexing uncertainty $u_{1}$ : The indexing uncertainty of the 5 RIRTs in NPL text is $0.5 \mathrm{mK}$, take $u_{1}=0.5 \mathrm{mK}$.

Difference uncertainty between ITS-90 and EPT-76, $u_{2}$ : The difference resolution between ITS-90 and EPT-76 is $0.1 \mathrm{mK}$ and the changes of the temperature difference between two adjacent integer point is less than $0.3 \mathrm{mK}$, take $u_{2}=0.2 \mathrm{mK}$.

Uncertainty caused by temperature scale conversion to ITS-90, $u_{3}$ : The RIRTs are converted to ITS-90 temperature scale and the electrical unit is changed. The least squares fitting calculation is introduced and the standard deviation to the power of 11 is less than $0.3 \mathrm{mK}$, take $u_{3}=$ $=0.2 \mathrm{mK}$.

Measurement uncertainty of F18 AC bridge, $u_{4}$ : The measurement accuracy of F18 AC bridge is $0.2 \mathrm{ppm}$, which equivalent $0.02 \mathrm{mK}$ in the lowest sensitivity temperature $27 \mathrm{~K}$, take $u_{4}=0.02 \mathrm{mK}$.

Divergence uncertainty of the F18 AC bridge readings, $u_{5}$ : When the temperature with the minimum bandwidth $0.02 \mathrm{~Hz}$ is measured, the divergence of the readings for F18 bridge is $0.3 \mathrm{mK}$, take $u_{5}=0.3 \mathrm{mK}$.

Uncertainty caused by standard resistance, $u_{6}$ : A $10 \Omega$ standard resistance is introduced when the F18 bridge is used to measure the temperature. Uncertainty given by test certification is $1 \mathrm{ppm}$, which is equivalent to $0.09 \mathrm{mK}$ in the lowest sensitivity temperature $27 \mathrm{~K}$, take $u_{6}=$ $=0.09 \mathrm{mK}$.

Uncertainty caused by temperature fluctuations, $u_{7}$ : The temperature fluctuations can achieve $0.6 \mathrm{mK}$ for the comparison measurement, take $u_{7}=0.6 \mathrm{mK}$.

Uncertainty caused by the self-heating effect of the RIRTs, $u_{8}$ : Due to the differences when the thermometer is installed, the maximum self-heating effect of the RIRT thermometer is $0.4 \mathrm{mK}$, take $u_{8}=0.4 \mathrm{mK}$. 
Uncertainty caused by temperature field inhomogeneities of the copper block, $u_{9}$ : The thermal diffusivity of constant copper block in low temperature is high, the uniformity of the temperature field is better than $0.1 \mathrm{mK}$, take $u_{9}=0.1 \mathrm{mK}$.

The standard uncertainty is synthesized based on the data above. The comparison measurement uncertainty of the RIRTs standard facility is $u=9.5 \mathrm{mK}$.

\section{Conclusion}

The control temperature level of 704 type cryostat could reach $0.4 \mathrm{mK} / 30 \mathrm{~min}$. The difference between the temperature value of the 5 RIRTs and the average is less than $1.1 \mathrm{mK}$. The comparison measurement uncertainty of the RIRTs reference standard facility is $0.95 \mathrm{mK}$.

The working temperature of the "National Temperature Working Standard Group of the ITS-90 at $1.2-24 \mathrm{~K}$ " is extended by 703 type cryostat to $0.65 \mathrm{~K}$ which is the lowest limit temperature of ITS-90.

The measurement value and its performance of this standard facility is proved accurate and reliable. This standard facility of RIRT could be served as national standard facility at $0.65-24 \mathrm{~K}$.
1. H.P. Thomas, Metrologia 27, 3 (1990).

2. P. Lin and L.M. Besley, Meas. Sci. Technol. 4, 1357 (1993).

3. R.L. Rusby and M. Kempson, Rev. Cen. Therm. 35, 338 (1996).

4. P. Mohandas, D.I. Head, and R.L. Rusby, Physica B 284, 2004 (2000).

5. R.L. Rusby, in: Temperature, its Measurement and Control in Science and Industry, American Institute of Physics, Pittsburgh (1972), p. 865.

6. R.L. Rusby, in: Temperature, its Measurement and Control in Science and Industry, American Institute of Physics, New York (1982), p. 829.

7. P. Lin, Y.Z. Mao, L.H. Yu, Q.G. Zhang, and C.S. Hong, Cryogenics 42, 443 (2002)

8. P. Lin, Y.Z. Mao, C.S. Hong, F. Pavese, I. Peroni, D. Head, and R.L. Rusby, in: Temperature, its Measurement and Control in Science and Industry, American Institute of Physics, New York (2003), p. 191.

9. P. Lin, Y.Z. Mao, C.S. Hong, Y. Yue, and Q.G. Zhang, Cryogenics 30, 432 (1990).

10. I. Peroni, F. Pavese, D. Ferri, P. Lin, Q.G. Zhang, and L.H. Yu, in: Proceedings of TEMPMEKO 2001, 8th International Symposium on Temperature and Thermal Measurements in Industry and Science, VDE Verlag GMBH, Berlin (2002), p. 391.

11. L. Yin, P. Lin, J.J. Zhao, and X. Qi, Metrol. Meas. Syst. 18, 13 (2011). 